\title{
PSEUDOSPECTRUM OF AN ELEMENT OF A BANACH ALGEBRA
}

\author{
ARUNDHATHI KRISHNAN AND S. H. KULKARNi
}

Abstract. The $\varepsilon$-pseudospectrum $\Lambda_{\varepsilon}(a)$ of an element $a$ of an arbitrary Banach algebra $A$ is studied. Its relationships with the spectrum and numerical range of $a$ are given. Characterizations of scalar, Hermitian and Hermitian idempotent elements by means of their pseudospectra are given. The stability of the pseudospectrum is discussed. It is shown that the pseudospectrum has no isolated points, and has a finite number of components, each containing an element of the spectrum of $a$. Suppose for some $\varepsilon>0$ and $a, b \in A, \Lambda_{\varepsilon}(a x)=\Lambda_{\varepsilon}(b x) \forall x \in A$. It is shown that $a=b$ if:

(i) $a$ is invertible.

(ii) $a$ is Hermitian idempotent.

(iii) $a$ is the product of a Hermitian idempotent and an invertible element.

(iv) $A$ is semisimple and $a$ is the product of an idempotent and an invertible element.

(v) $A=B(X)$ for a Banach space $X$.

(vi) $A$ is a $C^{*}$-algebra.

(vii) $A$ is a commutative semisimple Banach algebra.

Mathematics subject classification (2010): 47A10, 46H05, 47A12.

Keywords and phrases: Banach algebra, Hermitian, idempotent, numerical range, pseudospectrum, semisimple, spectrum.

\section{REFERENCES}

[1] J. Alaminos, J. Extremera, And A. R. Villena, Approximately spectrum-preserving maps, J. Funct. Anal. 261 (2011), pp. 233-266.

[2] Frank F. Bonsall and John DunCan, Numerical Ranges of Operators on Normed Spaces and of Elements of Normed Algebras, vol. 2 of London Math. Soc. Lecture Note Series, Cambridge University Press, Cambridge, 1971.

[3] Frank F. Bonsall and John Duncan, Complete Normed Algebras, Springer-Verlag, New York, 1973.

[4] AlbRecht BötTCHer, Pseudospectra and singular values of large convolution operators, J. Integral Equations Appl 6 (1994), pp. 267-301.

[5] Albrecht Böttcher and Hartmut Wolf, Spectral approximation for Segal-Bargmann space Toeplitz operators, Linear Operators, Banach Center Publications 38 (1997), pp. 25-48.

[6] MateJ BRešAR AND ŠPela ŠPENKo, Determining elements in Banach algebras through spectral properties, J. Math. Anal. Appl. 393 (2012), pp. 144-150.

[7] Jianlian Cui, Virginia Forstall, Chi-Kwong Li and Vincent Yannello, Properties and preservers of the pseudospectrum, Linear Algebra Appl. 436 (2012), pp. 316-325.

[8] Jianlian Cui, Chi-Kwong Li and Yiu-Tung Poon, Pseudospectra of special operators and pseudospectrum preservers, J. Math. Anal. Appl. 419 (2014), pp. 1261-1273.

[9] Mark Embree and Lloyd Nicolas Trefethen, Generalizing eigenvalue theorems to pseudospectra theorems, SIAM J. Sci. Comput. 23 (2001), pp. 583-590.

[10] Mark Embree and Lloyd Nicolas Trefethen, Pseudospectra Gateway, http://www.comlab.ox.ac.uk/pseudospectra. 
[11] Peter A. Fillmore, Notes on Operator Theory, Van Nostrand Reinhold Co., New York, 1970.

[12] J. Globevnik, Norm-constant analytic functions and equivalent norms, Illinois J. Math. 20 (1976), pp. 503-506.

[13] R. Hagen, S. Roch and B. Silbermann, $C *$-Algebras and Numerical Analysis, vol. 236 of Monographs and Textbooks in Pure and Applied Mathematics, Marcel Dekker Inc., New York, 2001.

[14] Paul R. Halmos, A Hilbert Space Problem Book, Springer-Verlag, New York, second ed., 1982.

[15] ANDERS C. HANSEN, On the solvability complexity index, the n-pseudospectrum and approximations of spectra of operators, J. Amer. Math. Soc. 24 (2011), pp. 81-124.

[16] T. Kato, Perturbation Theory for Linear Operators, Springer-Verlag, Berlin, 1995.

[17] S. H. KulKarni And D. Sukumar, The condition spectrum, Acta Sci. Math. (Szeged), 74 (2008), pp. 625-641.

[18] G. Krishna Kumar And S. H. KulKarni, Banach algebra techniques to compute spectra, pseudospectra and condition spectra of some block operators with continuous symbols, Ann. Funct. Anal. 6 (2015), pp. 148-169.

[19] George H. Orland, On a class of operators, Proc. Amer. Math. Soc. 15 (1964), pp. 75-79.

[20] A. PAZY, Semigroups of Linear Operators and Applications to Partial Differential Equations, vol. 44 of Applied Mathematical Sciences, Springer-Verlag, New York, 1983.

[21] C. R. Putnam, Operators satisfying a G1 condition, Pacific J. Math. 84 (1979), pp. 413-426.

[22] C. R. Putnam, Almost normal operators, their spectra and invariant subspaces, Bull. Amer. Math. Soc. 79 (1973), pp. 615-624.

[23] T. J. RANSFORD, Generalised spectra and analytic multivalued functions, J. Lond. Math. Soc. 2 (1984), pp. 306-322.

[24] Walter Rudin, Functional Analysis, International Series in Pure and Applied Mathematics, Tata McGraw-Hill, New Delhi, second ed., 2006.

[25] Eugene Shargorodsky, On the level sets of the resolvent norm of a linear operator, Bull. Lond. Math. Soc. 40 (2008), pp. 493-504.

[26] Eugene Shargorods Ky, On the definition of pseudospectra, Bull. Lond. Math. Soc. 41 (2009), pp. $524-534$.

[27] STANislaV ShKaRIN, Norm attaining operators and pseudospectrum, Integral Equations and Operator Theory 64 (2009), pp. 115-136.

[28] Allan M. Sinclair, The norm of a Hermitian element in a Banach algebra, Proc. Amer. Math. Soc., (1971), pp. 446-450.

[29] J. G. STAMPfLi, Hyponormal Operators and Spectral Density, Trans. Amer. Math. Soc. 117 (1965), pp. $469-476$.

[30] Lloyd Nicholas Trefethen and Mark Embree, Spectra and Pseudospectra: The Behavior of Nonnormal Matrices and Operators, Princeton University Press, Princeton, 2005. 